\section{MS24-P4 Structural and magentoelectric} study of triethylmethylammonium tetrachloroferrate(III)

Martina Vrankić ${ }^{1}$, Sanja Burazer ${ }^{1}$, Zvonko Jagličić ${ }^{2}$, Ana Šantić ${ }^{3}$, Jasminka Popović ${ }^{1}$

1. Division of Materials Physics, Ruđer Bošković Institute, Bijenička 54, 10000 Zagreb, Croatia

2. Institute of Mathematics, Physics and Mechanics, Jadranska 19 , SI-1000 Ljubljana, Slovenia

3. Division of Materials Chemistry, Ruđer Bošković Institute, Bijenička 54, 10000 Zagreb, Croatia

email: mvrankic@irb.hr

The novel organic-inorganic hybrids with a perovskite-type structure represent a potentially very attractive platform for interesting electrical and magnetic properties. In contrast to pure inorganic multiferroics, this class of materials exhibit new properties tuned by the nature of metal centers, organic cations and appropriate ligands. It was found that inorganic-organic hybride compounds containing tetrabromoferrate(III) anion exhibit the ferroelectric and magnetic phase transitions resulting in a strong magnetodielectric coupling at $\sim 360$ $\mathrm{K}$ [1]. Namely, the combination of disordered cation and magnetic transition metal ion results in formation of a magnetodielectric molecule-based functional material above room temperature.

A dark-yellow polycrystalline sample of a triethylmethylammonium tetrachloroferrate(III) compound was produced by the wet chemical synthesis. Detailed high temperature in-situ X-ray powder diffraction in the range from RT to $400 \mathrm{~K}$ was utilized in order to follow the temperature-induced structural changes. Title compound crystallizes in hexagonal space group P63mc at RT where the organic cationic part is completely disordered. Both the $\mathrm{FeBr}_{4}^{-}$anion and the organic cation lie on a hexagonal axis. It was found that structural phase transition of triethylmethylammonium tetrachloroferrate(III) was accompanied with changes in magnetic and electrical behaviour as shown in Figure 1. Since for most multiferroics, the magnetic phase transition temperature is much lower than the ferroelectric phase transition temperature, (finally resulting in a weak coupling between magnetic and ferroelectric orderings) this compound exhibiting transitions at similar temperatures shows potential for further investigations of magnetoelectric coupling.

1. Cai, H.-L., et al., Above-Room-Temperature Magnetodielectric Coupling in a Possible Molecule-Based Multiferroic: Triethylmethylammonium Tetrabromoferrate(III). Journal of the American Chemical Society, (2012)134, 18487-18490.
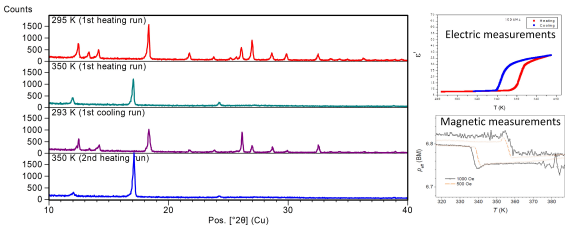

Figure 1. XRD, electric and magnetic measurements of triethylmethylammonium tetrachloroferrate(III) compound
Keywords: X-ray powder diffraction, organic-inorganic hybride compounds, phase transition 